\title{
Scared fitless: Context-dependent response of fear to loss of predators over evolutionary time in Drosophila melanogaster
}

\author{
Kyle H. Elliott ${ }^{a b \star}$, D. Ryan Norris ${ }^{b}$, Gustavo S. Betini ${ }^{\mathrm{b}}$, and Ian Dworkin ${ }^{\mathrm{c}}$ \\ ${ }^{a}$ Department of Natural Resource Sciences, McGill University, Ste-Anne-de-Bellevue, QC H9X 3V9, \\ Canada; ${ }^{b}$ Department of Integrative Biology, University of Guelph, Guelph, ON N1G 2W1, Canada; \\ ${ }^{\mathrm{c}}$ Department of Biology, McMaster University, Hamilton, ON L8S 4K1, Canada \\ *kyle.elliott@mcgill.ca
}

\begin{abstract}
Fear of predation can disappear rapidly in the absence of predators, as bolder individuals outcompete vigilant individuals for food and mates. To examine the evolution of fear in a seasonal environment, we exposed Drosophila melanogaster to mantid predators during the breeding season and the nonbreeding season, and compared these with a control. We compared three Drosophila lineages that were maintained in captivity for (1) 45 years without mantid predators, (2) $\sim 5$ years without mantid predators, and (3) $\sim 5$ years with mantid predators (predator-evolved). The presence of a predator during the non-breeding season caused reduced fecundity in the following breeding season, independent of the evolutionary lineage. However, the presence of a predator during reproduction caused offspring to emerge earlier, and this effect was more pronounced in the predator-evolved lineage. Thus, the fear response was related to evolutionary lineage only during the larval life stage, which is when foraging competition, and hence the cost of fear, may be highest. We present one of the first experimental demonstrations that emotion (fear) can evolve in response to environmental context.
\end{abstract}

Citation: Elliott KH, Norris DR, Betini GS, and Dworkin I. 20 I7. Scared fitless: Contextdependent response of fear to loss of predators over evolutionary time in Drosophila melanogaster. FACETS 2: 342-354. doi: 1 0.1 1 39/facets-2016-0075

Editor: Jeffrey Hutchings

Received: December 8, 2016

Accepted: February 17, 2017

Published: April II, 2017

Copyright: (C) 2017 Elliott et al. This work is licensed under a Creative Commons Attribution 4.0 International License (CC BY 4.0), which permits unrestricted use, distribution, and reproduction in any medium, provided the original author(s) and source are credited.

Published by: Canadian Science Publishing

Key words: carryover effects, experimental evolution, foraging-predation risk tradeoff, maternal effects, non-consumptive effects of predation, seasonality, evolution of instincts

\section{Introduction}

Non-consumptive effects of predators, including the effects of fear, can play an important role in animal ecology, influencing offspring development (Lardner 2000; Dahl and Peckarsky 2002; Benard 2004), adult fitness (Roitberg et al. 1979; Ylönen 1989; Dixon and Agarwala 1999; Gallagher et al. 2016), population dynamics (Lima 1998; Preisser et al. 2005; Creel et al. 2009), and even ecosystem function (Beckerman et al. 1997; Schmitz et al. 1997; Suraci et al. 2016). When risk of predation is high, animals spend more time being vigilant and less time foraging (Creel and Christianson 2008; Sheriff et al. 2009; Wirsing and Ripple 2011), leading to poorer body condition and physiological stress. Averaged across many studies, evidence suggests that the population-level effect of fear on prey mortality can be equivalent to the effect of direct predator consumption (Preisser and Bolnick 2008).

Although fear can have important ecological consequences, what is less clear is whether fear persists in the absence of predators over an evolutionary timescale. Understanding the differential evolution 
of naiveté might help assess the impact and success of invasive species, as the rapid evolution of fearfulness to novel, invasive predators would allow native prey to rapidly respond to such invasions (Sih et al. 2010). On the one hand, fear of predation diminishes in the absence of predators (Blumstein and Daniel 2005; Binz et al. 2014), with riskier individuals outcompeting less risky individuals for food and mates. Classic examples come from islands where naïve species are essentially fearless (Darwin 1839; Rödl et al. 2007). Indeed, Darwin's observation that animals on remote islands were unafraid of people was an impetus for his theory of natural selection, as he posited that escape behaviour was diminished where predators were rare due to the loss of costly escape responses in the absence of strong natural selection to maintain them (Darwin 1839; Cooper et al. 2014). On the other hand, many laboratory animals, such as rats, retain innate fear of predators, such as cats, after thousands of generations without predator exposure (Blanchard et al. 1990; Zangrossi and File 1992; Dielenberg et al. 2001). A major difference between laboratory and natural populations is that the latter are often limited by food, leading to costs associated with fearfulness that are not typically present in well-fed laboratory populations. Consequently, we might expect strong selection against fear in wild, but not laboratory, populations.

To examine the evolution of fear response, we investigated how cues from a mantid predator impact the fitness of Drosophila evolved with and without predators. Drosophila were exposed to the scent of a mantid predator either in a simulated breeding season, a simulated non-breeding season, or both. Fear responses are typically multimodal, and the effect of fear from the mantid is likely due to a combination of vibrations from the mantid, the scent of the mantid, and the scent of dead flies defecated by the mantid. Previously, we demonstrated that exposing naïve Drosophila to a predator (i) caused increased abdominal lifting, an antipredator behaviour in Drosophila (Parigi et al. 2014), (ii) reduced fecundity in the following breeding season, and (iii) quicker offspring development but reduced offspring adult mass (Betini et al. 2013; Elliott et al. 2016). The impact on offspring development was due to maternal effects from stressed mothers, as offspring did not emerge until the mantids were removed (Elliott et al. 2016). Thus, fear in the non-breeding season impacted fecundity in the breeding season and fear in mothers impacted offspring development in flies that had not been exposed to predators for many generations.

To understand the evolution of fear, we examined the two endpoints highlighted by our previous study (fecundity and development time) in lineages differing in the time since they had been exposed to predators. We compared three sets of lineages: one that had been purchased from a stock population centre (stock), a population derived from the wild and evolved without mantid predators (control-evolved), and a population derived from the same ancestral base population evolved with mantid predators (predator-evolved). We predicted that the responses (fecundity and emergence time) would be higher in the predator-evolved populations than in the control-evolved or stock populations because the latter two populations had not evolved to recognize mantid cues.

\section{Methods}

\section{Provenance of Drosophila lineages}

We used three lineages of Drosophila melanogaster. The stock population was from a population collected in Dahomey (now Benin) in 1970, which has since been maintained in a cage culture at $25^{\circ} \mathrm{C}$, and thus has not experienced predation for $\sim 900$ generations ( 45 years $\times 20$ generations/year). Prior to the experiment, the stock population was maintained on a sugar-yeast diet, with a $12 \mathrm{~L}: 12 \mathrm{D}$ light-dark cycle, and a $14 \mathrm{~d}$ cycle of non-overlapping generations with $3 \mathrm{~d}$ available for breeding each generation. The carryover effects and density dependence of the stock population to seasonality have previously been well quantified (Betini et al. 2013, 2015). Data from the stock population were 
compiled from the Data Appendix to Elliott et al. (2016), and those experiments took place 2 weeks before the experiments with the evolved populations.

The other two lineages were initially collected in 2010 at a vineyard in Fennville, Michigan (Parigi et al. 2014). This initial population (from over 500 pairs of D. melanogaster) was then split into three treatments (with four replicates each): control with no predators (control-evolved), mantid predator (predator-evolved), and spider predator (not used in this study). Both treatment groups used in this study were maintained in cages $\left(30 \mathrm{~cm}^{3}\right.$, BugDorm) with overlapping generations, and given cornmeal-molasses-yeast food in a $12 \mathrm{~L}: 12 \mathrm{D}$ light-dark cycle with $\sim 40 \%$ humidity at $\sim 25^{\circ} \mathrm{C}$. Two generations prior to the start of the experiment, five males and five females from each evolutionary lineage replicate were pooled together, switched to the same sugar-yeast diet, and placed on a $14 \mathrm{~d}$ cycle of non-overlapping generations with $3 \mathrm{~d}$ available for breeding each generation.

\section{Non-breeding and breeding seasons}

Many animals have distinct stages in their annual cycle (seasons) that correspond with environmental variation, principally food availability. Here, we consider a system with two seasons defined by whether an animal is breeding or not breeding (Betini et al. 2013), although the principles could be extended to more complex seasonality. Our system mimics several key elements of seasonality in the wild: (1) a breeding season that is much shorter than the non-breeding season; (2) flies that are not food restricted during the non-breeding season, but as the female has no source of protein and no laying medium, she therefore does not breed; and (3) the decline of fecundity with date (these points were reviewed by Betini et al. 2015; Elliott et al. 2016).

We simulated seasonality by creating distinct breeding and non-breeding seasons, following the protocol of Betini et al. (2013, 2015; Fig. 1). During the non-breeding season, adults were placed in an empty vial with access to $0.2 \mathrm{~mL}$ of $5 \%$ water-sugar solution for $4 \mathrm{~d}$, which prevented females from producing eggs. During the breeding season, flies were placed for $24 \mathrm{~h}$ into $10 \mathrm{~mL}$ of a dead yeastagar-sugar medium $\left(1000 \mathrm{~mL} \mathrm{H} \mathrm{H}_{2} \mathrm{O}, 100 \mathrm{~g}\right.$ sucrose, $50 \mathrm{~g}$ Fleischmann's yeast, $16 \mathrm{~g}$ agar, $8 \mathrm{~g}$ $\mathrm{C}_{4} \mathrm{H}_{4} \mathrm{KNaO}_{6}, 1 \mathrm{~g} \mathrm{KH}_{2} \mathrm{PO}_{4}, 0.5 \mathrm{~g} \mathrm{NaCl}, 0.5 \mathrm{~g} \mathrm{MgCl}_{2}, 0.5 \mathrm{~g} \mathrm{CaCl}_{2}$, and $\left.0.5 \mathrm{~g} \mathrm{Fe}_{2}\left(\mathrm{SO}_{4}\right)_{3}\right)$. Adults were then discarded, and the larvae were allowed to mature to adults. Flies were kept at $25^{\circ} \mathrm{C}$, with $12 \mathrm{~L}: 12 \mathrm{D}$ cycles, and humidity levels between $30 \%$ and $50 \%$. The same sized vial $(28 \mathrm{~mm} \times 95 \mathrm{~mm})$ was used for both seasons.

\section{Fear behavioural experiment}

We used Chinese praying mantids, Tenodera aridifolia sinensis (hereafter referred to as mantids) as predators. The behavioural response of Drosophila to Tenodera sinensis was studied in detail by Parigi et al. (2014), who observed that Drosophila increased abdominal bobbing in response to the mantid predator. There was a bimodal response in terms of the time spent grooming and the time spent walking, with most individuals spending more time grooming and walking, and others spending less time (Parigi et al. 2014). Rather than examining particular activities, we were interested in whether exposure to predators increased activity in our stock lines, potentially causing the reduction in energy stores at the end of the non-breeding season for mantidexposed flies.

We placed five recently emerged $(<48 \mathrm{~h}$ ) individual flies (four males and one female) into a $5 \mathrm{~mm}$ diameter glass vial, separated by a small piece of cotton from a dead mantid, a live mantid, or nothing (one control for each treatment), with $n=7$ replicates for dead mantid and live mantid treatments and $n=14$ replicates for the control treatment. The glass vials were placed in an activity monitor (DAM2, TriKinetics, Waltham, Massachusetts, USA) that recorded each time a fly broke a laser beam half way through the vial. The Drosophila were provided with sugar-water similar to our 


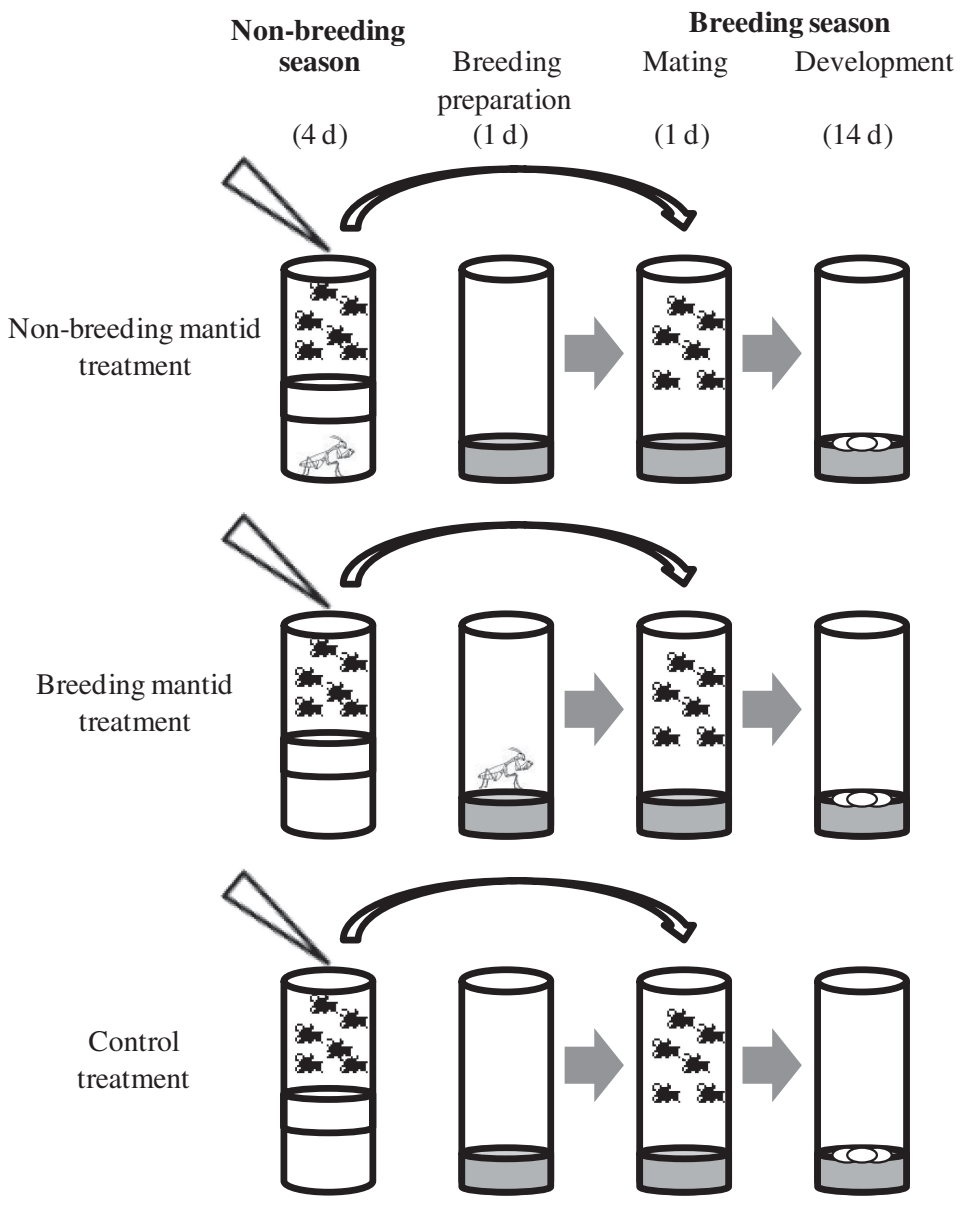

Fig. 1. Schematic diagram of the two treatments and control used in the study. All flies began in the non-breeding season (the tilted triangle at the top of the vial shows the sugar-water solution used to feed the flies during this period), and after $4 \mathrm{~d}$ were moved to the breeding season (the dark grey at the bottom of the vial represents the dead yeast-agar-sugar medium). The breeding season consisted of $24 \mathrm{~h}$ for mating and then $14 \mathrm{~d}$ for development of the larvae. For the non-breeding mantid treatment, mantids were placed at the bottom of the vial during the non-breeding season, and for the breeding mantid treatment, mantids were placed in breeding vials $1 \mathrm{~d}$ prior to the start of the breeding season and removed prior to addition of flies. Larvae emerged between $\mathrm{d} 8$ and 15 , with $>99 \%$ occurring between $\mathrm{d} 9$ and 13 (breeding season days numbered to include $1 \mathrm{~d}$ as eggs).

non-breeding season, and the activity patterns were monitored for $96 \mathrm{~h}$. We then used a general linear mixed model (with time and vial as random effects) to predict activity as a function of treatment.

\section{Fear carryover experiments}

We tested Drosophila with single first instar mantids that had been feeding on adult Drosophila for their entire lives prior to the start of the experiment. To control for age and body size, we used only individual adult Drosophila between 1 and $3 \mathrm{~d}$ old from parents bred at low density. To obtain these offspring, we selected males and females and placed 20 individuals (50:50 sex ratio) in separate $28 \mathrm{~mm} \times 95 \mathrm{~mm}$ vials with $10 \mathrm{~mL}$ of fresh food for $24 \mathrm{~h} \mathrm{(d} \mathrm{0).} \mathrm{It} \mathrm{takes} \mathrm{a} \mathrm{minimum} \mathrm{of} 8 \mathrm{~d}$ for offspring to develop. On d 9, we discarded all offspring and used only individuals that emerged between $\mathrm{d} 10$ and 12 . We then combined these offspring and randomly grouped 10 males and 
10 females into one of the three treatments: control $(n=15)$, non-breeding mantid $(n=15)$, and breeding mantid $(n=15)$.

All treatments began at the start of the non-breeding season and finished at the end of the breeding season. After $4 \mathrm{~d}$ in the non-breeding season, we moved the survivors to the breeding season, but controlled density by combining all survivors from each treatment at the end of the non-breeding period, and then placed eight males and eight females in each breeding vial. Fewer flies were used for the breeding season than the non-breeding season because $\sim 10 \%$ of flies died in the nonbreeding season.

For the non-breeding mantid treatment, immediately prior to the commencement of the nonbreeding season, we placed a first instar mantid at the bottom of the non-breeding season vial and placed a thin $(1 \mathrm{~cm}$ ) sponge between the mantid and the flies (Fig. 1). This prevented the mantid from ascending the vial and the flies from seeing the mantid. After $4 \mathrm{~d}$, the mantid and flies were removed and placed into the breeding season vial. The breeding season was identical for the non-breeding mantid and control treatments (Fig. 1).

For the breeding mantid treatment, the non-breeding season was identical to that of the control. After $4 \mathrm{~d}$, the flies were transferred into the breeding season vial. For the breeding mantid treatment, a sponge was placed in an empty non-breeding vial, and the mantid was added into the breeding season vial with fresh fly food $24 \mathrm{~h}$ before the survivors from the non-breeding season vial were transferred to the breeding season vial (Fig. 1).

To investigate the effects of the treatment on reproductive output, we counted the total number of offspring that emerged, which we termed fecundity. Over $90 \%$ of eggs hatched, and consequently the number of offspring emerged was very similar to, and highly correlated with, the number of eggs laid (Elliott et al. 2016). We also measured the proportion of individuals with early emergence, which was the proportion of individuals that emerged on $\mathrm{d} 9$. Variance in the proportion of individuals with early emergence among treatments was almost entirely due to variance in hatching time, and the proportion of individuals with early emergence was closely associated with hatching time but not with laying time (Elliott et al. 2016). Thus, the proportion of individuals with early emergence reflects the speed at which the Drosophila eggs hatched.

\section{Statistical analysis}

For the experiment with the stock population, we used general linear models, with post hoc Tukey's tests among all possible combinations with statistically significant model effects, to compare fecundity and the proportion of individuals with early emergence among treatments, lineages, and to assess their interactions. All analyses were conducted in R 3.2.0 (R Core Team 2016) with statistical significance set at $\alpha=0.05$.

The evolutionary background of the populations may have caused differences between the stock and evolved lineages beyond those associated with the predators. The stock flies were maintained for hundreds of generations under conditions that allowed them $3 \mathrm{~d}$ to reproduce before being terminated. The evolved lineages were derived more recently (2010) from a wild population and were maintained for $\sim 100$ generations under conditions that allowed them to reproduce indefinitely. As increased extrinsic mortality reduces longevity in Drosophila and increases fecundity (Rose and Charlesworth 1980; Zwaan et al. 1995; Stearns et al. 2000), it would be expected that the lineages with the shortest life spans would also have the highest fecundity (as observed). Similarly, the stock flies were kept under a regime that only allowed $3 \mathrm{~d}$ for breeding, whereas the breeding vials in the evolved populations were removed only after $25 \mathrm{~d}$, again potentially selecting for higher 
fecundity (as observed). Finally, the different diets may have played a role, and the evolved flies might have had lower fecundity because they were adjusting to a new diet. To account for those effects, we conducted separate linear models with only the evolved lineages. As those separate models did not change the statistical significance of any of our results, we relied principally on the omnibus model with the stock lineage included.

\section{Results}

Fecundity depended on the treatment $\left(F_{2,192}=61.2, p<0.0001\right)$ and evolutionary lineage $\left(F_{2,192}=190.5, p<0.0001\right)$ but not their interaction $\left(F_{3,192}=0.92, p=0.43\right)$. Fecundity was lower in the predator-evolved and control-evolved lineages than the stock lineage (Fig. 2a). In addition, compared with the control, fecundity in all three lineages was reduced with exposure to mantid cues during the non-breeding season. Furthermore, fecundity was also reduced by exposure to mantid cues during the breeding season for the predator-evolved and control-evolved lineages (Fig. 1a). When excluding the stock lineage, fecundity depended on treatment $\left(F_{2,84}=5.39, p=0.006\right)$ and evolutionary line (mantid vs. control, $\left.F_{1,84}=5.04, p=0.03\right)$ but not their interaction $\left(F_{2,84}=0.05\right.$, $p=0.95$ ). Thus, fecundity was always lower by a proportionately similar amount during the non-breeding mantid treatment but not the control or breeding mantid treatments, regardless of evolutionary line, but fecundity was also lower in the evolved lines than in the stock lines.

The proportion of individuals with early emergence depended on the treatment $\left(F_{2,192}=34.5\right.$, $p<0.0001)$ and the interaction between the treatment and lineage $\left(F_{3,192}=7.05, p=0.0002\right)$ but not lineage itself $\left(F_{2,192}=2.37, p=0.10\right)$. Within lineages, the proportion of individuals with early emergence was greater in the breeding treatment relative to the non-breeding and control treatments (Fig. 2b). For the predator manipulation during the breeding season, the proportion of individuals with early emergence was greater for the predator-evolved lineage relative to the stock and controlevolved lineages (Fig. 2b). The proportion of individuals with early emergence was also higher in the control treatment for the control-evolved and stock lineages than in the predator-evolved lineages, and during the non-breeding mantid treatment for the stock lineage relative to the predator-evolved lineage. When excluding the stock lineage, the proportion of individuals with early emergence depended on treatment $\left(F_{2,84}=43.7, p<0.00001\right)$ and the interaction between the treatment and line $\left(F_{2,84}=13.04, p=0.00001\right)$ but not the line itself $\left(F_{1,84}=0.38, p=0.54\right)$.

Activity was independent of the treatment for the entire $96 \mathrm{~h}\left(F_{3,25}=0.72, p=0.62\right)$ and when we only examined the first $24 \mathrm{~h}\left(F_{3,25}=0.55, p=0.65\right.$; Fig. 3$)$.

\section{Discussion}

Although we predicted that the carryover effect on both fecundity and development time would be higher in the predator-evolved populations than in the control-evolved or stock populations, we only observed a higher response in development time. We suggest that predator-evolved flies likely had greater earlier emergence than the other two groups in response to a perceived high cost to reduced foraging opportunities. In other words, this context-dependent (i.e., age-dependent) response could be associated with fearful behavior (i.e., reduced foraging) at early-life stages. In captivity, adult Drosophila are given food ad libitum. Under typical captive conditions, an adult Drosophila that is slightly more vigilant will still be able to feed to satiety and have no worse fecundity than one that is less vigilant, potentially explaining why we found no effect of lineage on fecundity and no difference in fecundity between the breeding mantid and control treatments. For instance, in the presence of mantids, the Drosophila showed similar levels of activity as during the absence of mantids. However, an earlier study demonstrated that they spent more time lifting their abdomens as a signal of vigilance (Parigi et al. 2014). Unless more time spent lifting led to higher energy expenditure, 

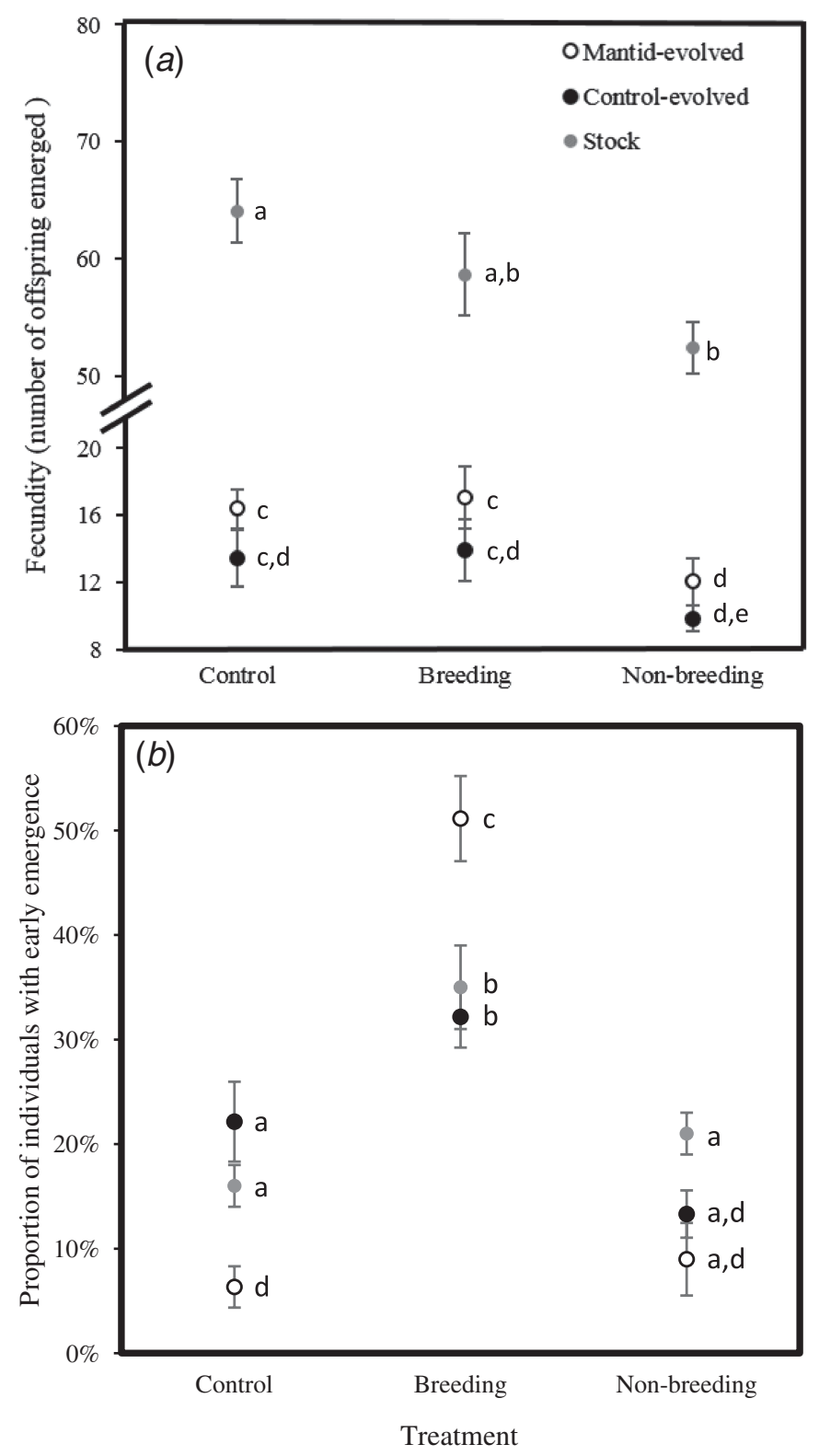

Fig. 2. (a) Total number (mean \pm SE) of offspring emerged, and (b) development time (mean \pm SE) for the stock (stock population captive for $\sim 900$ generations; grey symbols), control-evolved (wild population held captive within a cage without mantid predators for $\sim 1000$ generations; black symbols), and predator-evolved (wild population held captive within a cage with mantid predators for $\sim 1000$ generations; white symbols) populations. Drosophila lineages were exposed to three treatments: control (without mantid cues), breeding mantid (with mantid cues during the breeding season), and non-breeding mantid (with mantid cues during the non-breeding season). Treatments marked with the same lowercase letters within the figure panels are not significantly different from one another (post hoc Tukey's HSD test only on significant parameters within the ANOVA, $\alpha=0.05$ ). Data for the stock population are from the Data Appendix to Elliott et al. (2016).

similar levels of activity implies that the reduced body mass at the end of the non-breeding season is due to reduced foraging associated with increased vigilance, which would have little cost to a typical laboratory Drosophila fed ad libitum (unlike our non-breeding season). In contrast, larval 


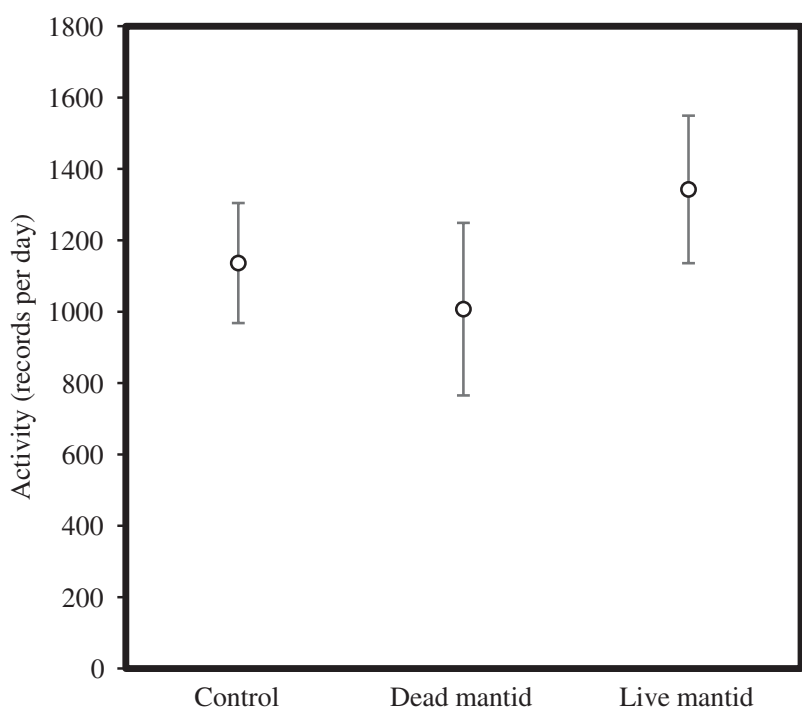

Fig. 3. Average activity per day (number of records of Drosophila breaking a laser at the mid-point of the vial as recorded by an automated activity recorder) over $96 \mathrm{~h}$ for four males and one female recently emerged Drosophila separated by a sponge from nothing (control; $n=14$ ), a dead mantid $(n=7)$, or a live mantid $(n=7)$.

Drosophila in captivity must eat voraciously to develop rapidly, attain maximum adult body size to gain mates or produce eggs (Mueller and Joshi 2000; Betini et al. 2013,2014), and avoid being cannibalized under high densities (Vijendravarma et al. 2013). Even a minor reduction in foraging efficiency due to false fear could have negative consequences, explaining why development time, and consequently adult body size (Elliott et al. 2016), were affected by exposure to predators. Given the differences in development time among lineages, differences in evolutionary background may explain why some studies find strong responses to fear and others only weak responses (Preisser et al. 2005; Preisser and Bolnick 2008).

The different evolutionary histories impacted the relative fecundity of the different lineages. The control lineage consisted of flies that had evolved for thousands of generations in an environment where they had only $3 \mathrm{~d}$ to reproduce before being destroyed (and the offspring used for the next generation). In contrast, the control-evolved and predator-evolved lineages were maintained in overlapping generations (fresh food added every $5 \mathrm{~d}$ but no flies experimentally culled). Therefore, the control lineage was potentially selected for high immediate fecundity compared with the evolved lineages. Similarly, the predator-evolved flies tended to have higher fecundity than the control-evolved flies. Those lineages with higher extrinsic mortality (e.g., predator-evolved) tend to evolve more rapid senescence and higher fecundity (Zwaan et al. 1995; Stearns et al. 2000). Similarly, predator-evolved flies evolved delayed emergence in the absence of predators compared with control-evolved flies. Possibly, because the larvae were not consumed by the mantids, they were selected to emerge later and larger (more fecund). Despite the very different evolutionary histories of our lines, we found that fear impacted fecundity and development time for all three lineages.

The foraging cost of being fearful explains why captive animals may retain fear responses to predators, whereas other animals, such as those on islands, often do not (Blanchard et al. 1990; Zangrossi and File 1992; Dielenberg et al. 2001; Rödl et al. 2007). Captive animals fed ad libitum, such as is common in Drosophila experimental cultures, face few fitness costs if they occasionally delay eating because they detect a scent reminiscent of a predator. In contrast, animals living on islands are limited by competition for food and exhibit classic K-selected traits such as low reproductive output and increased longevity (Tamarin 1977; Austad 1993). Thus, it is unsurprising that island species such as the Falkland Islands fox (Dusicyon australis) and dodo (Raphus cucullatus) evolved to have no fear 
of predators-an evolutionary trajectory that famously doomed these species once predators arrived. The evolution of naiveté also occurs in invertebrates, such as deaf moths living on bat-free islands (Fullard 1994) and zooplankton living in freshwater ponds that are eliminated by introduced predators (Cox and Lima 2006; Amundsen et al. 2009). We would predict that island species in which foraging competition during adulthood plays less of a role would retain fear in the absence of predators.

One potential criticism of our system is that the breeding season was shorter than the non-breeding season. However, this was purposeful as we attempted to create an ecologically relevant experimental system, and breeding seasons are shorter than non-breeding seasons for many wild animals (e.g., most migrating birds, and insects in northern temperate environments). Consequently, the flies in the breeding season were exposed to the predator for a shorter period than the flies in the non-breeding season, which may account for the differences in sensitivity to the predator treatments. A longer breeding season would be unlikely to change the impact of the predator on fecundity as $\sim 80 \%$ of eggs are laid within $6 \mathrm{~h}$ of exposure of the females to the medium (depending on when the females are added relative to light-dark cycles). In addition, larvae hatch after $\sim 22 \mathrm{~h}$, and as they grow they cannibalize eggs that are subsequently laid. The control treatment followed the same protocols as the other two treatments (see also the next section) but did not have a mantid added in either season (Fig. 1). An additional potential criticism is that the form of mantid cue was different between nonbreeding and breeding seasons, with the mantid being continually present during the non-breeding season, and only the scent remaining in the breeding season. However, the response in both cases was similar along a gradient of mantid sizes and presumably chemical cues associated with the mantids, meaning that the response to the cue was likely some form of threshold function (Elliott et al. 2016). Therefore, we must have been near the maximum threshold response in both cases, and the responses were thus apparently similar. Future studies should examine the relationship between the fear-like response and exposure to predator cues.

We provide evidence that fear impacted carryover effects across three different evolutionary contexts. Indeed, given that we measured a direct reduction in fecundity of $20 \%-25 \%$ and a likely reduction of subsequent fecundity of $\sim 10 \%$ (based on advancement in development time leading to reductions in adult size; Elliott et al. 2016), the total effect of fear on an equilibrium population size of $\sim 200$ flies in our environment was likely similar to the effect of direct mortality to predators ( $\sim 4 \mathrm{flies} / \mathrm{d}$; I. Dworkin, personal observation, 2014). Several recent studies have documented population declines of species in seasonal environments following increases in predator abundance (Ydenberg et al. 2004; Creel and Christianson 2008). We speculate that cross-seasonal carryover effects of fear on fecundity or offspring growth may play a role in such circumstances.

We add to the growing consensus that fear can impact fitness within and across seasons. Combined with our earlier experiments (Elliott et al. 2016), there is repeatable evidence that the fear of a predator in the non-breeding season leads to lower condition of individuals at the end of the non-breeding season, which then impacts subsequent fecundity. There is also clear evidence that the fear of a predator in the breeding season leads to earlier emergence and smaller flies. Thus, fear amplifies carryover effects across seasons and maternal effects from mother to offspring. Despite those fitness costs, the seasonal carryover effects are maintained for many generations in the absence of mantids, presumably because increased vigilance comes at little cost in typical well-fed lab environments (in contrast to our non-breeding season). In contrast, the reduced fitness costs associated with maternal effects presumably led to the evolution of fearlessness by larvae in the absence of mantids. The context-dependent results may explain why, for instance, some prey species remain resilient to invasive predators while others rapidly go extinct. Regardless, additional studies examining how different contexts impact the evolution of emotions, including fear, would be fruitful avenues for investigation. 


\section{Acknowledgements}

We thank B. Felipe, R. Scarfone, L. Teeger, A. Van Natto, and R. Wolach for assistance in the lab. This research was funded by both a postdoctoral fellowship to KHE and Discovery grants to DRN and ID from the Natural Sciences and Engineering Research Council, and a University of Guelph Research Chair to DRN.

\section{Author contributions}

Conceived and designed the study: KHE, DRN, GSB, ID. Performed the experiments/collected the data: KHE, DRN, GSB, ID. Analyzed and interpreted the data: KHE, DRN, GSB. Contributed resources: DRN, ID. Drafted or revised the manuscript: KHE, DRN, GSB, ID.

\section{Competing interests}

The authors have declared that no competing interests exist.

\section{Data accessibility statement}

All relevant data are within the paper.

\section{References}

Amundsen PA, Siwertsson A, Primicerio R, and Bøhn T. 2009. Long-term responses of zooplankton to invasion by a planktivorous fish in a subarctic watercourse. Freshwater Biology, 54: 24-34. doi:10.1111/j.1365-2427.2008.02088.x.

Austad SN. 1993. Retarded senescence in an insular population of Virginia opossums (Didelphis virginiana). Journal of Zoology, 229: 695-708. doi:10.1111/j.1469-7998.1993.tb02665.x.

Beckerman AP, Uriarte M, and Schmitz OJ. 1997. Experimental evidence for a behavior-mediated trophic cascade in a terrestrial food chain. Proceedings of the National Academy of Sciences, 94: 10735-10738. PMID:11038581. doi:10.1073/pnas.94.20.10735.

Benard MF. 2004. Predator-induced phenotypic plasticity in organisms with complex life histories. Annual Review of Ecology, Evolution, and Systematics, 35: 651-673. doi:10.1146/annurev. ecolsys.35.021004.112426.

Betini GS, Griswold CK, and Norris DR. 2013. Carry-over effects, sequential density dependence and the dynamics of populations in a seasonal environment. Proceedings of the Royal Society B: Biological Sciences, 280: 20130110. PMID:23516241. doi:10.1098/rspb.2013.0110.

Betini GS, Griswold CK, Prodan L, and Norris DR. 2014. Body size, carry-over effects and survival in a seasonal environment: consequences for population dynamics. Journal of Animal Ecology, 83: 1313-1321. PMID:24708450. doi:10.1111/1365-2656.12225.

Betini GS, Fitzpatrick MJ, and Norris DR. 2015. Experimental evidence for the effect of habitat loss on the dynamics of migratory networks. Ecology Letters, 18(6): 526-534. doi:10.1111/ele.12432.

Binz H, Bucher R, Entling MH, and Menzel F. 2014. Knowing the risk: crickets distinguish between spider predators of different size and commonness. Ethology, 120: 99-110. doi:10.1111/ eth.12183. 
Blanchard RJ, Blanchard DC, Rodgers J, and Weiss SM. 1990. The characterization and modelling of antipredator defensive behavior. Neuroscience \& Biobehavioral Reviews, 14: 463-472. PMID:2287483. doi:10.1016/S0149-7634(05)80069-7.

Blumstein DT, and Daniel JC. 2005. The loss of anti-predator behaviour following isolation on islands. Proceedings of the Royal Society of London B, 272: 1663-1668. PMID:16087420. doi:10.1098/rspb.2005.3147.

Cooper WE, Pyron RA, and Garland T Jr. 2014. Island tameness: living on islands reduces flight initiation distance. Proceedings of the Royal Society of London B, 281: 20133019. PMID:24403345. doi:10.1098/rspb.2013.3019.

Cox JG, and Lima SL. 2006. Naiveté and an aquatic-terrestrial dichotomy in the effects of introduced predators. Trends in Ecology \& Evolution, 21: 674-680. PMID:16879896. doi:10.1016/j. tree.2006.07.011.

Creel S, and Christianson D. 2008. Relationships between direct predation and risk effects. Trends in Ecology \& Evolution, 23: 194-201. PMID:18308423. doi:10.1016/j.tree.2007.12.004.

Creel S, Winnie JA, and Christianson D. 2009. Glucocorticoid stress hormones and the effect of predation risk on elk reproduction. Proceedings of the National Academy of Sciences, 106: 12388-12393. PMID:19617549. doi:10.1073/pnas.0902235106.

Dahl J, and Peckarsky BL. 2002. Induced morphological defenses in the wild: predator effects on a mayfly, Drunella coloradensis. Ecology, 83: 1620-1634. doi:10.1890/0012-9658(2002)083[1620: IMDITW]2.0.CO;2.

Darwin C. 1839. The voyage of the Beagle: journal of researches into the natural history and geology of the countries visited during the voyage of HMS Beagle round the world. Henry Colburn, London UK.

Dielenberg RA, Carrive P, and McGregor IS. 2001. The cardiovascular and behavioral response to cat odor in rats: unconditioned and conditioned effects. Brain Research, 897: 228-237. PMID:11282383. doi:10.1016/S0006-8993(01)02227-2.

Dixon AFG, and Agarwala BK. 1999. Ladybird-induced life-history changes in aphids. Proceedings of the Royal Society of London. Series B: Biological Sciences, 266: 1549-1553. doi:10.1098/ rspb.1999.0814.

Elliott KH, Betini GS, Norris DR, and Dworkin ID. 2016. Experimental evidence for within- and cross-seasonal effects of fear on survival and reproduction. Journal of Animal Ecology, 85: 507-515. PMID:26713420. doi:10.1111/1365-2656.12487.

Fullard JH. 1994. Auditory changes in noctuid moths endemic to a bat-free habitat. Journal of Evolutionary Biology, 7: 435-445. doi:10.1046/j.1420-9101.1994.7040435.x.

Gallagher AJ, Lawrence MJ, Jain-Schlaepfer SMR, Wilson ADM, and Cooke SJ. 2016. Avian predators transmit fear along the air-water interface influencing prey and their parental care. Canadian Journal of Zoology, 94: 863-870. doi:10.1139/cjz-2016-0164.

Lardner B. 2000. Morphological and life history responses to predators in larvae of seven anurans. Oikos, 88: 169-180. doi:10.1034/j.1600-0706.2000.880119.x. 
Lima S. 1998. Stress and decision making under the risk of predation: recent developments from behavioral, reproductive, and ecological perspectives. Advances in the Study of Behavior, 27: 215-290. doi:10.1016/S0065-3454(08)60366-6.

Mueller LD, and Joshi A. 2000. Stability in model populations (MPB-31). Princeton University Press, Princeton, New Jersey.

Parigi A, Porter C, Cermak M, Pitchers WR, and Dworkin I. 2014. How predator hunting-modes affect prey behavior: capture deterrence in Drosophila melanogaster. bioRxiv. doi:10.1101/010330.

Preisser EL, and Bolnick DI. 2008. The many faces of fear: comparing the pathways and impacts of nonconsumptive predator effects on prey populations. PLoS ONE, 3: e2465. PMID:18560575. doi:10.1371/journal.pone.0002465.

Preisser EL, Bolnick DI, and Benard MF. 2005. Scared to death? The effects of intimidation and consumption in predator-prey interactions. Ecology, 86: 501-509. doi:10.1890/04-0719

R Core Team. 2016. R: a language and environment for statistical computing. R Foundation for Statistical Computing, Vienna, Austria [online]: Available from R-project.org/.

Rödl T, Berger S, Romero LM, and Wikelski M. 2007. Tameness and stress physiology in a predatornaive island species confronted with novel predation threat. Proceedings of the Royal Society of London B, 274: 577-582. PMID:17476779. doi:10.1098/rspb.2006.3755.

Roitberg BD, Myers JH, and Frazer BD. 1979. The influence of predators on the movement of apterous pea aphids between plants. Journal of Animal Ecology, 48: 111-122. doi:10.2307/4104.

Rose M, and Charlesworth B. 1980. A test of evolutionary theories of senescence. Nature, 287: 141-142. doi:10.1038/287141a0.

Schmitz OJ, Beckerman AP, and O'Brien KM. 1997. Behaviorally mediated trophic cascades: effects of predation risk on food web interactions. Ecology, 78: 1388-1399. doi:10.1890/0012-9658(1997)078 [1388:BMTCEO]2.0.CO;2.

Sheriff MJ, Krebs CJ, and Boonstra R. 2009. The sensitive hare: sublethal effects of predator stress on reproduction in snowshoe hares. Journal of Animal Ecology, 78: 1249-1258. PMID:19426257. doi:10.1111/j.1365-2656.2009.01552.x.

Sih A, Bolnick DI, Luttbeg B, Orrock JL, Peacor SD, Pintor LM, et al. 2010. Predator-prey naïveté, antipredator behavior, and the ecology of predator invasions. Oikos, 119: 610-621. doi:10.1111/ j.1600-0706.2009.18039.x.

Stearns SC, Ackermann M, Doebeli M, and Kaiser M. 2000. Experimental evolution of aging, growth, and reproduction in fruitflies. Proceedings of the National Academy of Sciences, 97: 3309-3313. PMID:10716732. doi:10.1073/pnas.97.7.3309.

Suraci JP, Clinchy M, Dill LM, Roberts D, and Zanette LY. 2016. Fear of large carnivores causes a trophic cascade. Nature Communications, 7: 10698. PMID:26906881. doi:10.1038/ncomms10698.

Tamarin RH. 1977. Reproduction in the island beach vole, Microtus breweri, and the mainland meadow vole, Microtus pennsylvanicus, in southeastern Massachusetts. Journal of Mammalogy, 58: 536-548. PMID:336824. doi:10.2307/1380002. 
Vijendravarma RK, Narasimha S, and Kawecki TJ. 2013. Predatory cannibalism in Drosophila melanogaster larvae. Nature Communications, 4: 1789. PMID:23653201. doi:10.1038/ncomms2744.

Wirsing AJ, and Ripple WJ. 2011. A comparison of shark and wolf research reveals similar behavioral responses by prey. Frontiers in Ecology and the Environment, 9: 335-341. doi:10.1890/090226.

Ydenberg RC, Butler RW, Lank DB, Smith BD, and Ireland J. 2004. Western sandpipers have altered migration tactics as peregrine falcon populations have recovered. Proceedings of the Royal Society of London, Series B: Biological Sciences, 271: 1263-1269. PMID:15306350. doi:10.1098/ rspb.2004.2713.

Ylönen H. 1989. Weasels Mustela nivalis suppress reproduction in cyclic bank voles Clethrionomys glareolus. Oikos, 55: 138-140. doi:10.2307/3565886.

Zangrossi H, and File SE. 1992. Behavioral consequences in animal tests of anxiety and exploration of exposure to cat odor. Brain Research Bulletin, 29: 381-388. PMID:1393611. doi:10.1016/0361-9230 (92)90072-6.

Zwaan BJ, Bijlsma R, and Hoekstra RF. 1995. Direct selection on lifespan in Drosophila melanogaster. Evolution, 49: 649-659. doi:10.2307/2410318. 